Revta brasil. Bot., São Paulo, V.23, n.1, p.107-112, mar. 2000

\title{
Estrutura e dinâmica de uma população de Calophyllum brasiliense Camb. em floresta higrófila do sudeste do Brasil
}

\author{
MÁRCIA C.M. MARQUES ${ }^{1,3}$ e CARLOS ALFREDO JOLY ${ }^{2}$
}

(recebido em 9 de junho de 1999; aceito em 15 de dezembro de 1999)

\begin{abstract}
Population structure and dynamics of Calophyllum brasiliense Camb. in a swamp forest in Southeastern Brazil). Age and spatial structures of a population of Calophyllum brasiliense (Clusiaceae) were studied in a $3600 \mathrm{~m}^{2}$ area of a swamp forest in Brotas, São Paulo State. During the first census, 1658 plants were tagged and after one year this number increased to 1706. Age structure did not change over the period of study, with a higher number of seedlings (individuals $\leq 0.2 \mathrm{~m})$ and juveniles $(>0.2-2 \mathrm{~m})$ and a smaller number of subadults $(>2-10 \mathrm{~m})$ and adults $(>10 \mathrm{~m})$. Seedling $(29.7 \%)$ and juvenile $(5.3 \%)$ mortality was caused, mainly, by soil erosion and deposition during the rainy season, because the plants were buried. Subadult $(0.7 \%)$ and adult $(0 \%)$ mortality was very low. A large number of new seedlings was concentrated in the lower parts of the study site and in soil depressions where the water carried seeds which were deposited and germinated. Recruitment rate for seedlings was high $(48.1 \%)$, while for juvenile (7.3\%), subadult $(1.9 \%)$ and adult $(0 \%)$ were much lower. Plants of all classes, from seedlings to adults, presented a clustered distribution due to the topography of the study site, which facilitates seed accumulation, and to a higher number of seeds under the canopy of reproductive individuals. Fruit dispersion by bats or water, seed survival under hypoxic conditions, seedling tolerance to waterlogging, age structure with a significant predominance of seedlings, and population growth explain why $C$. brasiliense is the most abundant species in the forest studied and also in other similar forests in Southeastern Brazil.
\end{abstract}

Resumo - (Estrutura e dinâmica de uma população de Calophyllum brasiliense Camb. em floresta higrófila do sudeste do Brasil). As estruturas de tamanho e espacial de uma população de Calophyllum brasiliense (Clusiaceae) foram estudadas em uma área de $3600 \mathrm{~m}^{2}$ de floresta higrófila localizada em Brotas, SP. No primeiro censo, foram marcados 1658 indivíduos e, após um ano, este número havia aumentado para 1706. A estrutura de tamanho não mudou durante o período de estudo, com predomínio de plântulas (indivíduos $\leq 0,2 \mathrm{~m}$ ) e jovens $(>0,2-2 \mathrm{~m}$ ) e menor número de subadultos $(>2-10 \mathrm{~m})$ e adultos $(>10 \mathrm{~m})$. A mortalidade em plântulas $(29,7 \%)$ e jovens $(5,3 \%)$ foi bem maior que em subadultos $(0,7 \%)$ e adultos $(0 \%)$, e teve como causa principal o soterramento na época chuvosa. O maior número de plantas novas concentrou-se nos locais mais baixos da área, onde sementes trazidas pela água eram acumuladas. A taxa de recrutamento de plântulas foi alta $(48,1 \%)$, e a de jovens $(7,3 \%)$, subadultos $(1,9 \%)$ e adultos $(0 \%)$, bem menores. Plantas de todas as classes de tamanho apresentaram distribuição espacial agregada, devido à topografia do terreno, que favorece o encharcamento do solo e o acúmulo de sementes, e à presença de sementes sob os adultos reprodutivos. Dispersão de frutos por morcegos ou pela água, capacidade de sobrevivência em condições hipóxicas, estrutura de tamanho com predomínio de plântulas e jovens e crescimento da população, são os principais fatores que determinam C. brasiliense como a espécie de maior importância na floresta estudada e também em outras florestas semelhantes do sudeste do Brasil.

Key words - Swamp forest, population dynamics, demography, Calophyllum brasiliense

\section{Introdução}

1. Departamento de Botânica, SCB, Universidade Federal do Paraná, Caixa Postal 19031, 81531-970 Curitiba, PR, Brasil.

2. Departamento de Botânica, IB, Universidade Estadual de Campinas, Caixa Postal 6109, 13083970 Campinas, SP, Brasil.

3. Autor para correspondência: mmarques@bio. ufpr.br
A manutenção da diversidade e da organização das comunidades tropicais podem ser discutidas a partir de dados populacionais (Connell et al. 1984). As estruturas das populações de plantas resultam da ação de fatores bióticos e abióticos sobre seus membros atuais e ancestrais, que afetam o arranjo espacial e as estruturas etária e genética de seus componentes (Hutchings 1997). Estes mesmos fatores provocam, 
também, mudanças temporais e espaciais no número de plantas das populações. Quando se quantificam os nascimentos e as mortes, é possível responder questões, tais como a coexistência de espécies raras e comuns, bem como relacionar os processos responsáveis pela flutuação deste número na comunidade (Watkinson 1997), além de fornecer informações sobre a capacidade de regeneração e a ocorrência de perturbações em determinado local (Harper 1977).

As florestas higrófilas, ao contrário das demais florestas ciliares, são formações de distribuição restrita, caracterizadas por apresentarem-se inundadas durante praticamente todo o ano. A diversidade é baixa, pois poucas espécies conseguem sobreviver nestes ambientes eminentemente anóxicos (LeitãoFilho 1982). Uma destas espécies é Calophyllum brasiliense Camb. (Clusiaceae) que, além de ocorrer nestas florestas higrófilas, distribui-se, também, nas florestas atlântica, amazônica e em determinados locais do domínio do cerrado, desde a América Central até o litoral norte catarinense, sempre condicionada à condição de umidade do solo (Reitz et al. 1978, Oliveira-Filho \& Ratter 1995).

Apesar dos estudos sobre a demografia de espécies que ocorrem em florestas ciliares serem escassos (Leite \& Salomão 1992), podem fornecer informações importantes sobre o funcionamento destes ambientes e auxiliar projetos de recuperação e manejo. No presente trabalho, foi estudada uma população de Calophyllum brasiliense, em uma floresta higrófila do sudeste do Brasil, com o objetivo de descrever a estrutura de tamanho, a sobrevivência e o recrutamento das plantas, relacionando-os às condições hídricas locais.

\section{Material e métodos}

Caracterização da área de estudo e da espécie - O trabalho foi realizado em um fragmento de floresta higrófila com 13 ha, localizado na bacia do rio Jacaré-Pepira, em Brotas, SP ( $22^{\circ} 16^{\prime} \mathrm{S}, 48^{\circ} 06^{\prime} \mathrm{W}, 470 \mathrm{~m}$ altitude). A área é um local com várias nascentes, cuja água conflui para formar dois córregos, conferindo alta umidade ao solo durante todo o ano. O clima regional é classificado como tropical subquente úmido, com precipitação média anual de $1421 \mathrm{~mm}$, estação seca em julho-agosto e temperatura média anual de $22,1{ }^{\circ} \mathrm{C}$ (Marques 1994).

Calophyllum brasiliense é a espécie arbórea mais importante da floresta, principalmente em termos de dominância relativa (S. Menezes-Silva et al., dados não publicados). Na área, floresce nos meses de outubronovembro e frutifica, a partir de janeiro, por um período de 10 meses, estando as flores e frutos disponíveis na estação úmida (Marques 1994). Seus frutos são drupas e podem ser dispersos pela água ou por morcegos (Philostomidae).

Levantamento das características físicas da área -Uma área de $3600 \mathrm{~m}^{2}(45 \times 80 \mathrm{~m})$ no centro da floresta higrófila foi dividida em 144 parcelas de 5 x $5 \mathrm{~m}$. Neste local, estimouse a saturação hídrica de cada parcela, através do levantamento topográfico, que foi feito pelo método de quadrículas, com a cota estabelecida de $662 \mathrm{~m}$ e interpolação das curvas de nível a cada $20 \mathrm{~cm}$. A radiação fotossinteticamente ativa (PAR) disponível para a vegetação foi, também, medida com um quantum/radiômetro/ fotômetro LI-189 (Li-cor), seguindo metodologia semelhante à proposta por Brower \& Zar (1984). As leituras foram feitas em dias claros, entre 10:00 e 14:00 h, a $1 \mathrm{~m}$ de distância do solo, em cinco pontos estabelecidos em cada parcela (LI) e também em dois pontos, em área fora da floresta (LE). No total, foram registrados dados de 720 pontos da área, que foram transformados em valores de transmissividade ( $\mathrm{T}$ ), que é expressa em porcentagem, pela fórmula T = LI/LE.100. Para efeito de comparação, calcularam-se as médias dos valores das cotas altimétricas que passavam em cada parcela e da transmissividade dos cinco pontos de leitura.

Levantamento das plantas - Nos períodos de maio a julho de 1991 e de 1992, épocas coincidentes com o final da frutificação da espécie na área (M.C.M. Marques \& E.A. Fischer, dados não publicados), todos os indivíduos de $C$. brasiliense foram marcados e tiveram sua altura e diâmetro da base registrados. No segundo censo, os indivíduos com altura inferior a $2 \mathrm{~m}$ foram medidos, sendo também anotados o número de indivíduos novos incorporados na população (regenerantes) e o número de mortos.

Com base nas características morfológicas das plantas (Gatsuk et al. 1980), associadas às alturas das mesmas, foi feita uma divisão por classes de tamanho que refletisse os estádios de desenvolvimento de $C$. brasiliense. Desta maneira, a presença de cotilédones (a germinação é criptocotilar hipogeal), em alguns dos indivíduos, serviram para determinar a altura máxima para plântula $(0,2 \mathrm{~m})$; os jovens não apresentavam mais cotilédones, porém o corpo da planta encontrava-se em início de crescimento secundário (>0,2-2 m); os sub-adultos eram indivíduos em estádio pré-reprodutivo ( $>2-10 \mathrm{~m}$ ) e os adultos, os reprodutivos (> $10 \mathrm{~m})$.

A estrutura de tamanho da população nos dois anos foi comparada por um teste KolmogorovSmirnov (Sokal \& Rohlf 1981). Para o período de um ano, calculou-se a taxa de crescimento da popu- 
lação e as taxas de mortalidade (Brower \& Zar 1984) e de recrutamento $\left(\mathrm{n}^{\mathrm{o}}\right.$ de plantas que mudaram de classe de tamanho $/ \mathrm{n}^{\mathrm{o}}$ de plantas no primeiro censo) para cada classe de tamanho. Neste trabalho, o termo recrutamento refere-se tanto à incorporação de novas plântulas na população como à transição de plantas de uma classe para a outra.

Para a avaliação da distribuição espacial da população, os indivíduos amostrados foram mapeados. Na análise do padrão espacial, foi utilizado o índice de dispersão de Morisita (IM). O padrão diferente do aleatório foi verificado por um teste $\chi^{2}$ (Brower \& Zar 1984).

Analisou-se a existência de correlação de Spearman entre os dados físicos da área e a densidade de plantas e entre a densidade de indivíduos das diferentes classes. No caso da transmissividade, os valores foram convertidos pela fórmula arcoseno.proporção $^{1 / 2}$. Em todos os testes, a existência de um padrão biológico foi considerado para $\alpha=0,05$.

\section{Resultados}

Características físicas da área de estudo - A diferença entre os locais mais baixos e os mais elevados da área variou entre 2 e $3 \mathrm{~m}$, mas, nas proximidades do leito dos riachos, as cotas encontravam-se a até $5 \mathrm{~m}$ abaixo das áreas mais elevadas. A diferença topográfica ocasiona um mosaico de áreas inundadas e drenadas, que se alternam durante o ano. A PAR média que chega a $1 \mathrm{~m}$ do solo variou entre 0 e $32 \%$, entre as parcelas (figura 1). A maior parte da área é sombreada e os valores de transmissividade encontravam-se entre 0 e $4 \%$ (figura 1). Os locais com maior incidência de luz, são poucos (figura 1) e restringem-se a um grupo de parcelas onde outrora houve corte de algumas árvores.

Estrutura de tamanho da população - Na distribuição em classes de tamanho dos indivíduos amostrados no primeiro censo, observa-se uma predominância de plantas nas classes menores (plântulas e jovens), com progressiva queda nas classes maiores (tabela 1). No recenso, estas distribuições se mantiveram $(\mathrm{D}=$ $0,025, \mathrm{p}>0,05)$.

Mortalidade e recrutamento - A mortalidade total (192 indivíduos) foi sobreposta pela natalidade (238 plântulas), o que resultou em um crescimento da população, de 1658 para 1704 indivíduos (taxa de

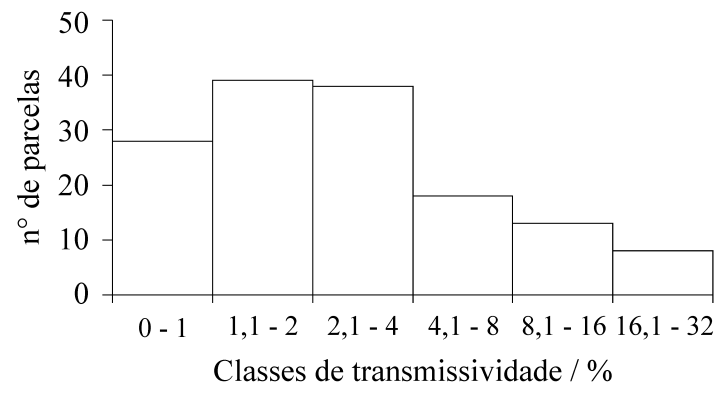

Figura 1. Distribuição de frequiência em classes de transmissividade, nas 144 parcelas da floresta higrófila em Brotas, SP.

crescimento $=0,0267$ ). A mortalidade, que foi maior entre plântulas e diminuiu nas classes posteriores, foi ocasionada principalmente pelo soterramento que os indivíduos menores sofreram devido ao carreamento de matéria orgânica e solo pela água dos córregos, na estação chuvosa. $\mathrm{O}$ recrutamento foi maior entre as plântulas, seguido pelos jovens e subadultos. $\mathrm{O}$ crescimento dos indivíduos parece ser lento, pois as taxas de recrutamento, principalmente entre jovens, subadultos e adultos foram baixas (figura 2).

Distribuição espacial dos indivíduos - Foi possível reconhecer diferentes intensidades de agrupamentos de plantas, nas classes consideradas. O IM foi de 1,46 para plântulas $\left(\chi^{2}=399, \mathrm{p}<0,05\right)$, de 2,46 para jovens $\left(\chi^{2}=1291, \mathrm{p}<0,05\right)$, de 1,85 para subadultos $\left(\chi^{2}=401, \mathrm{p}<0,05\right)$ e de 1,81 para adultos $\left(\chi^{2}=196\right.$, $\mathrm{p}<0,05)$, indicando a distribuição agregada dos indivíduos de todas as classes de tamanho, principalmente jovens e subadultos.

Tabela 1. Distribuição de freqüência em classes de tamanho, da população de Calophyllum brasiliense, numa floresta higrófila em Brotas, SP, no primeiro e segundo censos.

\begin{tabular}{lcc}
\hline Classes de tamanho & \multicolumn{2}{c}{ Ano } \\
& 1991 & 1992 \\
\hline Plântula & 495 & 550 \\
Jovem & 809 & 787 \\
Subadulto & 288 & 301 \\
Adulto & 66 & 66 \\
\hline Total & 1658 & 1704 \\
\hline
\end{tabular}




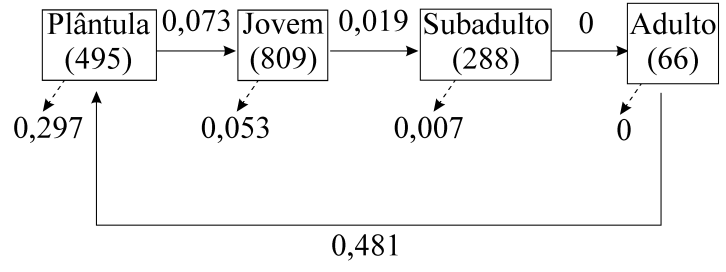

Figura 2. Esquema com as taxas de recrutamento $(\longrightarrow) \mathrm{e}$ mortalidade $1---\rightarrow$ ) para cada classe de tamanho de Calophyllum brasiliense, numa floresta higrófila em Brotas, SP. (n) é o número de indivíduos no primeiro censo.

Relações de densidade - A densidade média ( \pm desvio padrão) da população de $C$. brasiliense em Brotas, no primeiro censo, foi 4617 ( \pm 4119$)$ indivíduos.ha- ${ }^{-1}$ e, no segundo, 4717 ( \pm 4121$)$ indivíduos.ha ${ }^{-1}$. Entre os parâmetros abióticos analisados, a posição topográfica teve maior correlação com a densidade de plantas do que a PAR. Parcelas situadas em menores altitudes e, conseqüentemente mais úmidas, foram as que apresentaram o maior número de adultos $(\mathrm{r}=0,41, \mathrm{p}<0,05)$ e regenerantes $(r=0,22, p<0,05)$. A PAR que chega a $1 \mathrm{~m}$ do solo não se correlacionou significativamente com a distribuição de plantas, em nenhuma classe de tamanho e nem com o número de mortas ou regenerantes.

As densidades de plântulas e jovens $(r=0,26$, $\mathrm{p}<0,05)$, plântulas e subadultos $(\mathrm{r}=0,23, \mathrm{p}<0,05)$ e jovens e subadultos $(r=0,28, p<0,05)$ mantiveram uma correlação positiva. A mortalidade foi maior em parcelas com maior número de subadultos $(\mathrm{r}=0,24$, $\mathrm{p}<0,05)$ e a regeneração em parcelas com mais jovens $(r=0,22, p<0,05)$ e subadultos $(r=0,28$, $\mathrm{p}<0,05)$. Parcelas com maior número de plantas novas também apresentaram mais plantas mortas ( $\mathrm{r}$ $=0,48, \mathrm{p}<0,05)$.

\section{Discussão}

A tolerância de uma espécie à inundação é condicionada pela sobrevivência da semente e pela manutenção do crescimento das plantas em condições anóxicas (Kozlowski 1984). Calophyllum brasiliense é uma espécie capaz de germinar após vários meses de submersão, embora, enquanto inundadas, as sementes não germinem; suas plântulas crescem normalmente, tanto em solo inundado quanto drenado Marques \& Joly 2000). Estas carac- terísticas fazem com que a espécie seja muito frequiente em vários ambientes ribeirinhos do sudeste do Brasil e também em outros tipos de ambientes neotropicais onde o solo é permanentemente ou periodicamente inundado (Scarano et al. 1997). Em Brotas, as características topográficas locais promovem alterações de declividade, que favorecem o acúmulo de sementes dispersas pela água na época mais úmida e a posterior germinação nestes sítios, o que explica a correlação existente entre altas densidades de regenerantes e menores altitudes. Produção maior de frutos em árvores inundadas (E.A. Fischer \& F.A.M. Santos, dados não publicados) pode, também, favorecer esta situação. O curso dos riachos se altera a cada ano, mas os locais mais baixos são, também, aqueles onde há maior densidade de adultos, o que sugere que, com o passar do tempo, o recrutamento de plantas de várias classes é favorecido nos locais inundados. Apesar de condições de sombreamento terem sido relacionadas com baixo recrutamento em outra população de $C$. brasiliense (Fischer 1990), em Brotas a radiação não está correlacionada com a mortalidade e nem com a densidade de plantas.

A estrutura de tamanho de uma população pode demonstrar a sua forma de regeneração do passado e do presente (Knowles \& Grant 1983, Agren \& Zackrisson 1990). A maioria das populações de plantas mostra uma distribuição com predominância de indivíduos nas classes menores e poucos nas maiores (Solbrig 1981). Este padrão pode ser explicado como sendo uma conseqüência da permanência de plântulas nesta classe, por longos períodos, até ocorrer a queda de uma árvore ou uma alta mortalidade, que ocasionariam um crescimento rápido posterior (Webb et al. 1972). C. brasiliense mostra este tipo de distribuição, que é um reflexo da ocorrência de maiores taxas de recrutamento e mortalidade nas classes menores, com progressiva redução nas maiores. Estas taxas, iguais a zero entre subadultos e adultos, são esperadas, pois o período de um ano provavelmente não seja suficiente para se detectar alterações estruturais nas últimas classes de tamanho de populações de espécies arbóreas.

Segundo Weiner (1985), a interferência de indivíduos de classes maiores sobre as menores e, conseqüentemente, a estrutura das populações são alteradas com a densidade. No entanto, comparando 
a população de $C$. brasiliense em Brotas com a de outros locais no sudeste do Brasil (Fischer 1990, Scarano et al. 1997), as populações apresentaram densidades distintas mas estruturas bastante semelhantes. Isto sugere que fatores relacionados às condições físicas de cada local e às interações da espécie com a biota devam ser mais importantes na determinação de sua abundância do que as relações existentes dentro da própria população. No entanto, a manutenção de uma mesma estrutura na população de $C$. brasiliense, em Brotas, durante o período de estudo, somada ao fato de a mesma estar crescendo, provavelmente contribuem para os elevados valores de densidade e importância da espécie dentro da comunidade.

A maioria das populações de espécies tropicais tem a mortalidade concentrada nas classes menores (Solbrig 1981) e as causas são a presença de patógenos ou herbívoros e a competição entre plântulas ou entre plântulas e indivíduos adultos (Augspurger 1983, Swaine et al. 1987, Howe 1990). Em Brotas, a maioria dos indivíduos que morreram eram plântulas, mas a predação foi baixa e teve papel secundário. O soterramento das plantas devido à variação do leito dos riachos foi a principal causa de morte na população estudada e pode ser um fator importante nas florestas ciliares em geral. Como a densidade de adultos não teve relação com a densidade de indivíduos de outras classes, mas a correlação foi positiva entre plântulas, jovens e subadultos, é provável que, logo após o estabelecimento, os caules de jovens e subadultos reduzam a velocidade da correnteza e facilitem a deposição de material em suspensão ao seu redor, evitando o soterramento.

A distribuição espacial dos indivíduos e as distâncias de recrutamento não são constantes com o tempo e é o acúmulo destas modificações que determinarão o padrão espacial dos adultos (Augspurger 1983, Clark \& Clark 1984, Sterner et al. 1986). Todas as classes de tamanho da população de $C$. brasiliense mostraram-se agregadas. Segundo Hubbell (1979, 1980), esta distribuição é comum em espécies que apresentam "pontos de origem”, os quais são locais onde há altas densidades populacionais rodeados por áreas de menor densidade. No caso de $C$. brasiliense, os pontos de origem devem ser representados pelos agrupamentos de sementes e plântulas nas depressões, abaixo dos poleiros dos morcegos ou mesmo sob os indivíduos adultos. A partir deste ponto, as plantas sofrem mortalidade, devido ao soterramento e à densidade e, com o tempo, estes fatores irão determinar a sobrevivência de poucos indivíduos, que ainda estarão distribuídos de forma agregada.

A manutenção das florestas ciliares e de outros ambientes neotropicais sujeitos à saturação hídrica do solo depende das estratégias que permitam a sobrevivência da espécie (Joly 1991). Talauma ovata, por exemplo, espécie que ocorre e foi estudada nesta mesma área, combina a dispersão em época seca com mecanismos de tolerância à inundação das plântulas, na estação úmida, para garantir a sobrevivência (Lobo \& Joly 1995). O sucesso adaptativo de $C$. brasiliense em ambientes inundados se deve à dispersão, que ocorre principalmente na estação úmida, à existência de hidrocoria e à tolerância das plantas à anoxia. A capacidade de ocupação de novos ambientes é explicada, em parte, pelo fato das sementes serem dispersas por morcegos, muitos dos quais com uma ampla área de ocorrência, o que contribui para maior distribuição da espécie (M.C.M. Marques \& E.A. Fischer, dados não publicados). Variações ambientais, sucesso ou não na reprodução e mudanças no nível de competição interespecífica proporcionam diferenças nas populações de locais distintos.

Agradecimentos - Este trabalho é parte da Dissertação de Mestrado da primeira autora. Agradecemos ao Dr. Flavio Antonio Maës dos Santos, pela leitura prévia do manuscrito; à Patrícia Carneiro Lobo, pelo auxílio no trabalho de campo; ao Dr. George Shepherd, pela leitura do abstract; a Erich A. Fischer e Flavio A. M. dos Santos por permitirem o uso de parte de seus dados; ao CNPq, pela cessão de bolsa à primeira autora e às instituições financiadoras do projeto: Prefeitura Municipal de Brotas (SP), Consórcio Intermunicipal para a Preservação da Bacia do Jacaré-Pepira, IBAMA (Instituto Brasileiro do Meio Ambiente e Desenvolvimento), CNPq (Conselho de Desenvolvimento Científico e Tecnológico), FINEP (Financiadora de Estudos e Projetos), FAPESP (Fundação de Amparo à Pesquisa do Estado de São Paulo), FAEP/UNICAMP (Fundo de Apoio ao Ensino e à Pesquisa da UNICAMP), Conservation International, UNESCO/MAB e Grupo Pão de Açúcar. 


\section{Referências bibliográficas}

AGREN, J. \& ZACKRISSON, O. 1990. Age and size structure of Pinus sylvestris populations on mires in central and northern Sweden. Journal of Ecology 78:1049-1062.

AUGSPURGER, C.K. 1983. Seed dispersal of tropical tree Platypodium elegans, and the escape of its seedlings from fungal pathogens. Journal of Ecology 71:759-771.

BROWER, J.E. \& ZAR, J.H. 1984. Field \& laboratory methods for general ecology. W.C. Brown Publishers, Boston.

CLARK, D.A. \& CLARK, D.B. 1984. Spacing dynamics of a tropical rain forest tree: evaluation of the JanzenConnell model. American Naturalist 124:769-788.

CONNELL, J.H., TRACEY, J.G. \& WEBB, L.J. 1984. Compensatory recruitment, growth, and mortality as factors maintaining rain forest tree diversity. Ecological Monographs 54:141-164.

FISCHER, E.A. 1990. Distribuição de frequiência de classes de tamanho e estratégia reprodutiva de Calophyllum brasiliense Camb. (Guttiferae) em mata ciliar na Estação Ecológica Estadual Juréia-Itatins, São Paulo. In Anais do II Simpósio de ecossistemas da costa sul e sudeste brasileira, Publicação da ACIESP 71-1, v.I, p.337-347.

GATSUK, L.E., SMIRNOVA, O.V., VORONTZOVA, I., ZAUGOLNOVA, L.B. \& ZHUKOVA, L.A. 1980. Age states of plants of various growth forms: a review. Journal of Ecology 68:675-696.

HARPER, J.L. 1977. Population biology of plants. Academic Press, London.

HOWE, H.F. 1990. Survival and growth of juvenile Virola surinamensis in Panama: effects of herbivory and canopy closure. Journal of Tropical Ecology 6:259-280.

HUBBELL, S.P. 1979. Tree dispersion, abundance, and diversity in a tropical dry forest. Science 203:12991309.

HUBBELL, S.P. 1980. Seed predation and the coexistence of tree species in tropical forests. Oikos 35:214-229.

HUTCHINGS, M.J. 1997. The structure of plant populations. In Plant ecology (M.J. Crawley, ed.). Blackwell Science, Oxford, p.325-358.

JOLY, C.A. 1991. Flooding tolerance in tropical trees. In Plant life under oxygen deprivation: ecology, physiology and biochemistry (M.B. Jackson, D.D. Davies $\&$ H. Lambers, eds.) SBP Academic Publishing, The Hague, p.23-34.

KNOWLES, P. \& GRANT, M. 1983. Age and size structure analyses of engelmann spruce, ponderosa pine, lodgepole pine, and limber pine in Colorado. Ecology 64:1-9.
KOZLOWSKI, T.T. 1984. Responses of woody plants to flooding. In Flooding and plant growth (T.T. Kozlowski, ed.). Academic Press, London, p.129-163.

LEITÃO-FILHO, H.F. 1982. Aspectos taxonômicos das florestas do Estado de São Paulo. Silvicultura em São Paulo 16A:197-206.

LEITE, A.M.C. \& SALOMÃO, A.N. 1992. Estrutura populacional de regenerantes de Copaíba (Copaifera langsdorfii Desf.) em mata ciliar do Distrito Federal. Acta Botanica Brasilica 6:123-134.

LOBO, P.C. \& JOLY, C.A. 1995. Mecanismos de tolerância à inundação de plantas de Talauma ovata St. Hil. (Magnoliaceae), uma espécie típica de matas de brejo. Revista Brasileira de Botânica 18:177-183.

MARQUES, M.C.M. 1994. Estudos auto-ecológicos do guanandi (Calophyllum brasiliense Camb. Clusiaceae) em uma mata ciliar do município de Brotas, SP. Dissertação de mestrado, Universidade Estadual de Campinas, Campinas.

MARQUES, M.C.M \& JOLY, C.A. 2000. Germinação e crescimento de Calophyllum brasiliense (clusiaceae), uma espécie típica de florestas inundadas. Acta Botanica Brasilica 14 (no prelo)

OLIVEIRA-FILHO, A.T. \& RATTER, J.A. 1995. A study of the origin of central brazilian forests by the analysis of plant species distribution patterns. Edinburg Journal of Botany 52:141-194.

REITZ, R., KLEIN, R.M. \& REIS, A. 1978. Projeto madeira de Santa Catarina. Sellowia 28-30:218-224.

SCARANO, F.R., RIBEIRO, K.T., MORAES, L.F.D. \& LIMA, H.C. 1997. Plant establishment on flooded and unflooded patches of a freshwater swamp forest in Southeastern Brazil. Journal of Tropical Ecology 14:793-803.

SOKAL, R.R. \& ROHLF, J.E. 1981. Biometry. Freeman, San Francisco.

SOLBRIG, O.T. 1981. Studies on the population biology of the genus Viola. II. The effect of plant size on fitness in Viola sororia. Evolution 35:1080-1093.

STERNER, R.W., RIBIC, C.A. \& SCHATZ, G.E. 1986. Testing for life historical changes in spatial patterns of four tropical tree species. Journal of Ecology 74:621-633.

SWAINE, M.D., LIEBERMAN, D. \& PUTZ, F.E. 1987. The dynamics of tree population in tropical forest: a review. Journal of Tropical Ecology 3:359-366.

WATKINSON, A.R. 1997. Plant population dynamics. In Plant ecology (M.J. Crawley, ed.). Blackwell Science, Oxford, p.359-400.

WEBB, L.J., TRACEY, J.G. \& WILLIAMS, W.T. 1972. Regeneration and pattern in the subtropical rain forest. Journal of Ecology 60:675-695.

WEINER, J. 1985. Size hierarchies in experimental populations of annual plants. Ecology 66:743-752. 